\title{
Fast light solitons in resonant media
}

\author{
G. S. Agarwal and T. N. Dey* \\ Department of Physics, Oklahoma State University, Stillwater, Oklahoma 74078, USA
}

(Received 22 August 2006; published 5 April 2007)

\begin{abstract}
Using the structure of the Maxwell-Schrödinger equations in two-level systems in the transient domain, we derive both single-soliton and multisoliton $(2 n \pi$ pulse) solutions associated with fast light in resonant media. The analytical solutions enable us to understand the nature of fast light, and should open up new investigations of fast light in the form of localized solutions in a variety of media. We also derive the fast-soliton pair in three-level systems with gain.
\end{abstract}

DOI: 10.1103/PhysRevA.75.043806

PACS number(s): 42.65.Tg, 42.50.Md

Since the classic work of Hau et al. on ultraslow light [1], there have been a large number of papers demonstrating the ultraslow propagation of light in gaseous media [2-5], solidstate media $[6,7]$, and two-level saturable absorbers $[8,9]$. Work on gain media [10] and media with reverse saturation $[11,12]$ has led to fast light. However, much of the work has been done for media with relaxation fast compared to the pulse widths. An important question arises-is the existence of slow and fast light characteristic of a medium with strong relaxation, or can one obtain such light under transient conditions, i.e., when the pulse widths are much smaller than the relaxation times? A recent paper [13] has demonstrated fast light in an inverted two-level medium and has given an explicit soliton solution for such light. Remarkably, a sech solution was obtained for pulse propagation in gain media.

Clader et al. [13] obtained a significant pulse advancement of more than many pulse widths, which is quite remarkable compared to the previous steady-state results [10]. The analytical solutions are especially useful for understanding fast light, which is rather counterintuitive. Clearly, it is important to understand if there is any relation between solitons $[14,15]$ in an absorbing medium and those in a gain medium. Such a relation would enable us to understand not only single solitons, but also multisolitons, associated with slow and fast light. In this paper, we derive results for fastlight soliton solutions in both two- and three-level systems that can be studied experimentally in a variety of media. These solutions enable one to study fast soliton-soliton collisions as well as $2 n \pi$ pulses in two-level media. We mention further that all the analytical solutions have been checked against results obtained from direct numerical integration of the Maxwell-Schrödinger equations. Our results also show explicitly the backward propagation of fast light, which has attracted considerable attention recently [16].

We consider the propagation of an optical pulse in the direction $\mathrm{z}$ in a medium of two-level atoms. The electric field of the incident pulse can be written as

$$
\vec{E}(z, t)=\frac{1}{2}\left[\overrightarrow{\mathcal{E}}(z, t) e^{i(k z-\omega t)}+\text { c.c. }\right],
$$

where $c k=\omega$, and $\overrightarrow{\mathcal{E}}(z, t)$ is the slowly varying envelope of the pulse. We also note that, earlier, several authors [17]

\footnotetext{
*Electronic address: tarak.dey@gmail.com
}

obtained sech solutions outside the slowly-varying-envelope approximation in media with either gain or absorption. These are solutions without a carrier wave and are expected to occur at very large power levels of the order of $10^{14} \mathrm{~W} / \mathrm{cm}$. Kaplan and Shkolnikov also noticed that such solutions (called electromagnetic bubbles) for inverted media would have velocity greater than $c$. However, most experiments deal with fields with a well-defined carrier wave, and therefore we concentrate on solutions in the slowly-varyingenvelope approximation. In the rotating-wave approximation, the equations for the probability amplitudes $\mathcal{C}_{i}(i=1,2)$ of the atomic levels are

$$
\begin{gathered}
\frac{\partial}{\partial t} \mathcal{C}_{1}=-i \Delta \mathcal{C}_{1}+i \frac{\Omega}{2} \mathcal{C}_{2}, \\
\frac{\partial}{\partial t} \mathcal{C}_{2}=i \frac{\Omega^{*}}{2} \mathcal{C}_{1},
\end{gathered}
$$

where we denote the excited-state amplitude by $\mathcal{C}_{1}$. In Eq. (2), $\Delta$ is the detuning $\omega_{12}-\omega$ and $\Omega$ is the Rabi frequency defined by $\Omega=\vec{d}_{12} \cdot \overrightarrow{\mathcal{E}}(z, t) / \hbar$, where $\vec{d}_{12}$ is the dipole matrix element. In the slowly-varying-envelope approximation, we obtain the evolution of the equation for the Rabi frequency of the incident field:

$$
\left(\frac{\partial}{\partial z}+\frac{1}{c} \frac{\partial}{\partial t}\right) \Omega=i \mu \mathcal{C}_{2}^{*} \mathcal{C}_{1},
$$

where the coupling constant $\mu$ is given by $\mu$ $=4 n \pi \omega\left|d_{12}\right|^{2} / c \hbar$ with $n$ as the density of atoms in the medium. As usual, we work in the traveling frame of reference: $\tau=t-z / c, \zeta=z$. The set of coupled Maxwell-Schrödinger equations (2) and (3) has been extensively studied $[14,15]$. Much of the existing literature is for the case of absorbers. For a gain medium, we have obtained a general result by using the formal structure of the Maxwell-Schrödinger equations and proved the following result: The solution $\Omega_{G}$ for a gain medium can be obtained from the solution $\Omega_{A}$ for the absorber by using the transformation

$$
\Omega_{G}(\tau, \zeta: \mu, \Delta)=\Omega_{A}^{*}(\tau, \zeta:-\mu,-\Delta) .
$$

We base our argument on the fact that, under conditions of negligible relaxation, there is essentially no difference in 


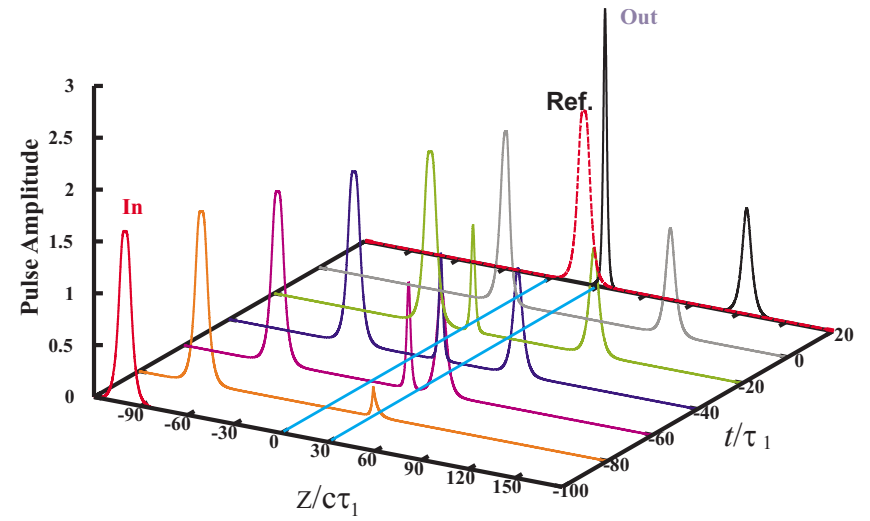

(a)

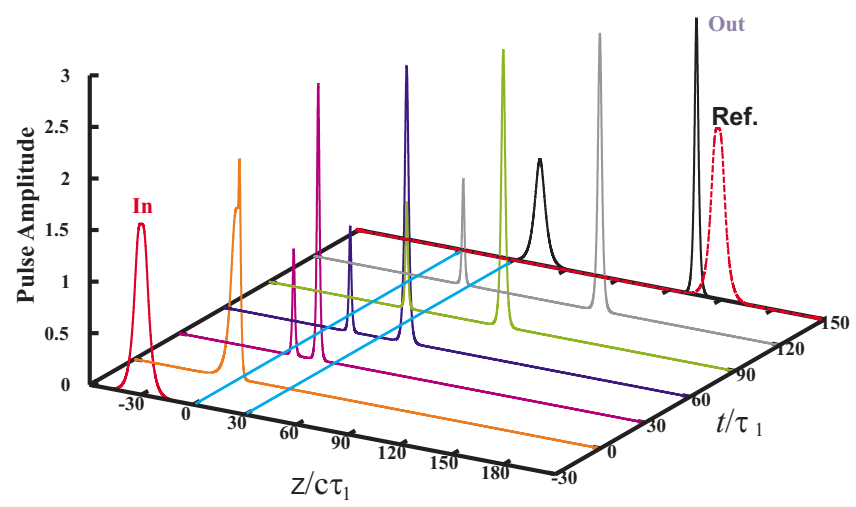

(b)

FIG. 1. (Color online) Multisoliton amplitude in the medium plotted against the spatial length of the medium at different propagation times. The medium occupies the region $z / c \tau_{1}=0$ to $z / c \tau_{1}$ $=30$. The propagation of a $4 \pi$ pulse in a gain (absorbing) medium is given by (a) [(b)]. (b) A two-soliton propagates in a two-level absorbing medium. The parameters used in the above graphs are $\tau_{1} / \tau_{2}=(3-\sqrt{5}) / 2$ and $\mu c \tau_{1}^{2}=0.5$.

what we label as $|1\rangle$ or $|2\rangle$. In order to see (4), we consider transformation of (2) and (3) via

$$
\mathcal{F}_{2}=\mathcal{C}_{1} e^{i \Delta t}, \quad \mathcal{F}_{1}=\mathcal{C}_{2} e^{i \Delta t}, \quad \widetilde{\Omega}=\Omega^{*}
$$

then the equations for $\mathcal{F}$ and $\widetilde{\Omega}$ are the same as Eqs. (2) and (3) provided we use $\mu \rightarrow-\mu$ and $\Delta \rightarrow-\Delta$. Using (4) we obtain fast-soliton as well as fast-multisoliton solutions in gain media. Let us first consider the well-known single-soliton solution in an absorber:

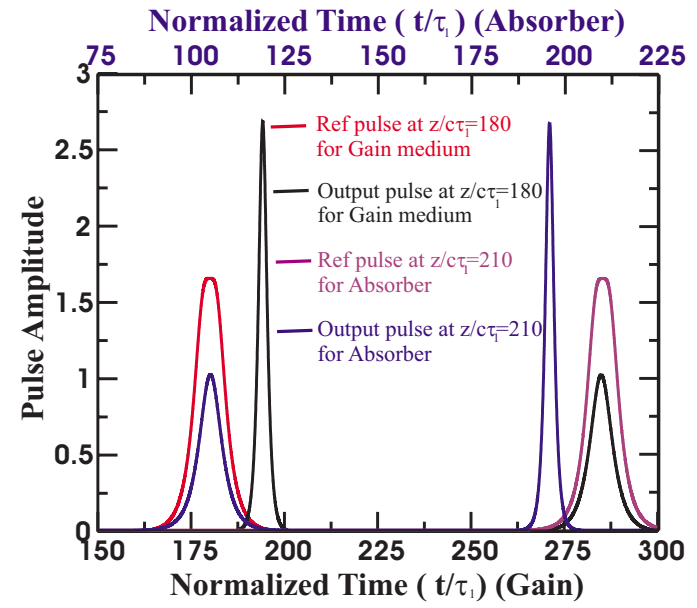

FIG. 2. (Color online) Amplitude of $4 \pi$ pulse at the exit of the medium plotted against the normalized time for $\mu c \tau_{1}^{2}=0.5$ and the ratio of pulse widths $\tau_{1} / \tau_{2}=(3-\sqrt{5}) / 2$. The output pulses for gain and absorbing media are double peaked.

$$
\begin{gathered}
\Omega_{A}(\tau, \zeta, \mu, \Delta)=A \operatorname{sech}\left(K \zeta-\frac{\tau}{\tau_{1}}\right) e^{i \beta \zeta}, \\
K=\frac{\mu \tau_{2} / 2}{1+\left(\tau_{2} \Delta\right)^{2}}, \quad \beta=K \Delta \tau_{2}, \quad A=\frac{2}{\sqrt{\tau_{2} \tau_{1}}} .
\end{gathered}
$$

Therefore, according to (4) the solution for the gain medium would be

$$
\Omega_{G}=A \operatorname{sech}\left(-K \zeta-\frac{\tau}{\tau_{1}}\right) e^{-i \beta \zeta}
$$

where $K$ and $\beta$ remain defined by (6). It should be borne in mind that (6) and (7) are valid inside the medium. The solutions outside the medium would have the form $A \operatorname{sech}\left(\tau / \tau_{1}\right.$ $+\theta) e^{-i \beta \zeta}$, where $\theta$ is a phase shift to be determined by matching it with (7) at the boundaries: $\Omega_{G}(\zeta<0)=A \operatorname{sech}\left(\tau / \tau_{1}\right)$; $\Omega_{G}(\zeta>L)=A \operatorname{sech}\left(\tau / \tau_{1}+K L\right) e^{-i \beta L}$. These solutions were obtained in Ref. [13] by using Bäcklund transformations [15].

The general result (6) also enables us to investigate a whole class of other solitons in gain media. It also enables us to investigate soliton-soliton interactions in gain media. For the two-soliton ( $4 \pi$ pulse) in gain media, we have found the result, using the formulation of Lamb [15] and Eq. (4) for the envelope,

$$
\Omega_{G}=A \frac{\frac{2}{\tau_{1}} \operatorname{sech}\left(\frac{t}{\tau_{1}}-\frac{z}{\tau_{1} V_{1}}\right)+\frac{2}{\tau_{2}} \operatorname{sech}\left(\frac{t}{\tau_{2}}-\frac{z}{\tau_{2} V_{2}}\right)}{1-B\left[\tanh \left(\frac{t}{\tau_{1}}-\frac{z}{\tau_{1} V_{1}}\right) \tanh \left(\frac{t}{\tau_{2}}-\frac{z}{\tau_{2} V_{2}}\right)-\operatorname{sech}\left(\frac{t}{\tau_{1}}-\frac{z}{\tau_{1} V_{1}}\right) \operatorname{sech}\left(\frac{t}{\tau_{2}}-\frac{z}{\tau_{2} V_{2}}\right)\right]},
$$




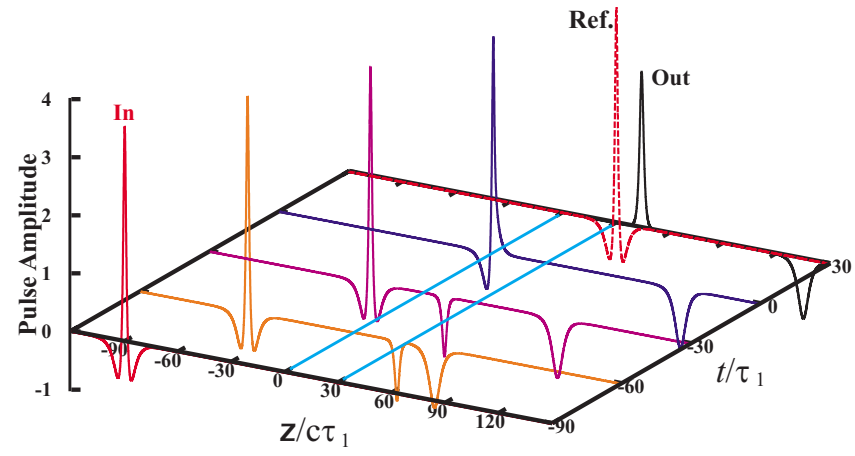

(a)

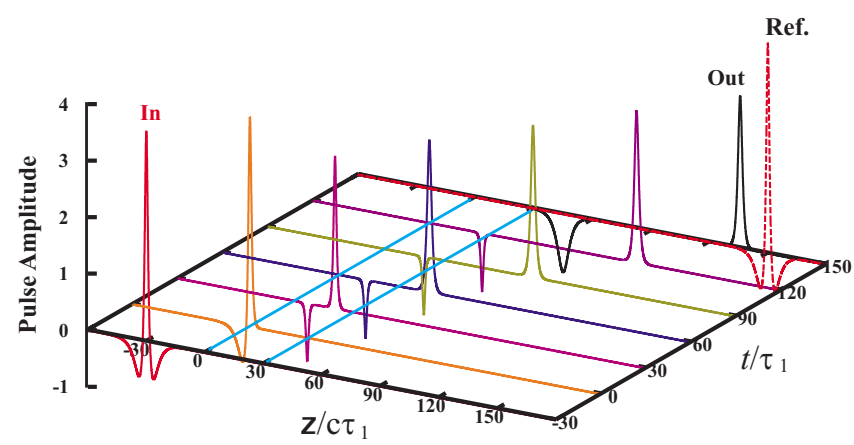

(b)

FIG. 3. (Color online) (a) and (b) show the spatial evolution of the $0 \pi$-soliton amplitude at different propagation times for a gain and an absorptive medium, respectively. The parameters are chosen as $\tau_{1} / \tau_{2}=(3-\sqrt{5}) / 2$ and $\mu c \tau_{1}^{2}=0.5$.

where we have chosen zero detuning and where

$$
A=\frac{\left(\tau_{2}^{2}-\tau_{1}^{2}\right)}{\left(\tau_{2}^{2}+\tau_{1}^{2}\right)}, \quad B=\frac{\left(2 \tau_{1} \tau_{2}\right)}{\left(\tau_{2}^{2}+\tau_{1}^{2}\right)}, \quad \frac{1}{V_{i}}=\frac{\left(1-\mu c \tau_{i}^{2}\right)}{c}
$$

These solutions have to be connected to the outside solitons in the same manner as single solitons. For $z>L$, the solution is given by (8) with $\left(t / \tau_{i}-z / \tau_{i} V_{i}\right)$ replaced by $\left(t / \tau_{i}-z / c \tau_{i}\right.$ $\left.+L \mu \tau_{i}\right)$. We show in Fig. 1 the nature of the $4 \pi$ solution in a gain medium, and for comparison we also show the $4 \pi$ solution in an absorbing medium. In the case of the gain medium, we see the appearance of a small peak at the other boundary, much before the pulse has a chance to enter the medium. This peak then moves toward the left, and at the same time a peak starts exiting the medium. This continues until the peak moving leftward catches up with the one entering the medium. This behavior in the gain medium is quite distinct from the behavior in a noninverted medium. We note that the backward propagation in a gain medium in an optical fiber has been recently reported under steady-state conditions [16]. The pulse advancement (delay) due to the gain (absorptive) medium is clearly seen in Fig. 2, although it is not easy to quantify the advancement or delay due to the doubledpeak nature of the output [18].

We can similarly study other types of fast-soliton solutions. For example, the result for a $0 \pi$ pulse in a gain medium can be obtained from Eq. (8) by changing the + sign to - in the numerator and the - sign to + between the two (a)

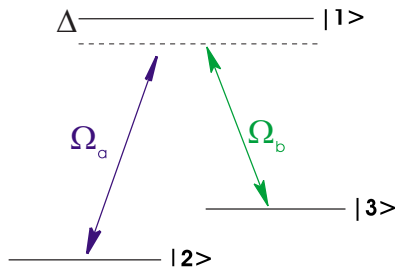

(b)

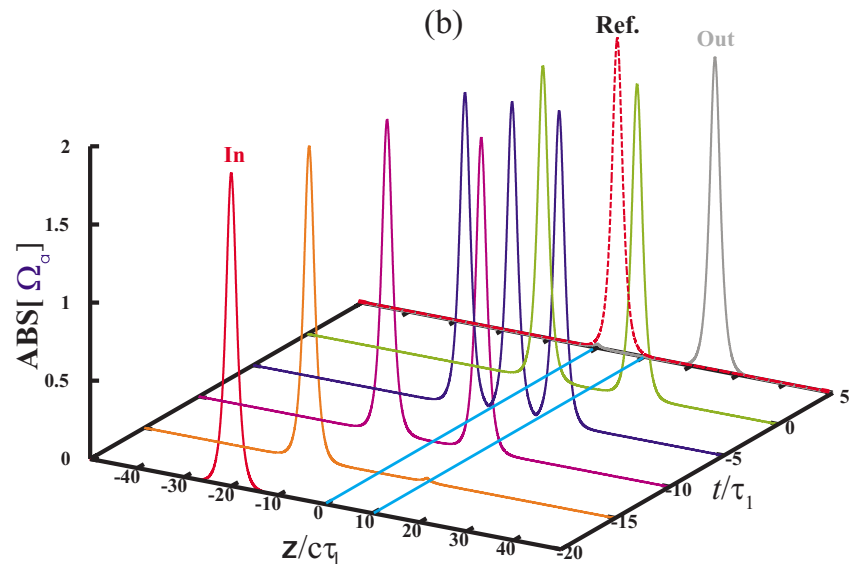

FIG. 4. (Color online) (a) Schematic diagram of a $\Lambda$-type atomic medium. The sech pulses $\Omega_{a}$ and $\Omega_{b}$ are in two-photon resonance with overall detuning $\Delta$ in the $\Lambda$-type atomic medium.(b) Spatiotemporal evolution of single soliton as given in Eq. (11) in a $\Lambda$-type atomic medium for $\mu c \tau_{1}^{2}=20$ and $\Delta=2 / \tau_{1}$.

terms in square brackets of the denominator. Figure 3 shows the spatiotemporal evolution of a $0 \pi$ pulse in media with gain and absorption, respectively.

We can also obtain soliton solutions for fast light in multilevel systems. Consider, for example, a $\Lambda$ system with two fields that satisfy the two-photon resonance condition, as shown in Fig. 4(a). The basic equations are given by

$$
\begin{gathered}
\frac{\partial}{\partial \tau} \mathcal{C}_{1}=-i \Delta \mathcal{C}_{1}+i \frac{\Omega_{a}}{2} \mathcal{C}_{2}+i \frac{\Omega_{b}}{2} \mathcal{C}_{3}, \\
\frac{\partial}{\partial \tau} \mathcal{C}_{2}=i \frac{\Omega_{a}^{*}}{2} \mathcal{C}_{1}, \quad \frac{\partial}{\partial \tau} \mathcal{C}_{3}=i \frac{\Omega_{b}^{*}}{2} \mathcal{C}_{1}, \\
\frac{\partial}{\partial \zeta} \Omega_{a}=i \mu \mathcal{C}_{1}^{*} \mathcal{C}_{2}, \quad \frac{\partial}{\partial \zeta} \Omega_{b}=i \mu \mathcal{C}_{1}^{*} \mathcal{C}_{3} .
\end{gathered}
$$

Initially, the atomic population is in the state $|1\rangle$. We can prove a relation between the solutions of a $\Lambda$ system with gain and the solutions for a $\mathrm{V}$ system with population in the ground state. The solutions for the latter case are available from the work of Rahman and Eberly [19]. We find the following result for the solitons in a $\Lambda$ system with population in the excited state:

$$
\Omega_{a}=A_{a} \operatorname{sech}\left(-K \zeta-\frac{\tau}{\tau_{1}}\right) e^{-i \beta \zeta}, \quad \Omega_{b}=\frac{A_{b}}{A_{a}} \Omega_{a},
$$




$$
K=\frac{\mu \tau_{1} / 2}{1+\left(\tau_{1} \Delta\right)^{2}}, \quad \beta=K \tau_{1} \Delta, \quad A_{a}^{2}+A_{b}^{2}=\frac{4}{\tau_{1}^{2}}
$$

The nature of the solution is similar to that in a two-level medium. This is because of the symmetries inherent in Eq. (10) and the initial condition. Figure 4(b) shows the very prominent generation of a background soliton, which then merges with the input soliton. The parameters used are for room-temperature Doppler-broadened ${ }^{87} \mathrm{Rb}$ vapor with excited-state lifetime $\mathrm{T}_{2}=28 \mathrm{~ns}$, density $n \approx 10^{11}$ atom $/ \mathrm{cm}^{3}$, $\lambda=794 \mathrm{~nm}$, and pulse duration $\tau_{1}=0.1 \mathrm{~ns}$. The range is also consistent with results for other atomic vapors provided the pulse width is many picoseconds [20].

In conclusion, we have discussed the existence of fast solitons, both single and multiple solitons, in a two-level gain medium. The analytical solutions clearly show how a backward wave can be generated. We also presented result for fast solitons in $\Lambda$ systems with gain. The results can be generalized to include inhomogeneous broadening, although we have not included it for simplicity [21], as well as to deal with other classes of systems such as $\mathrm{N}$ systems [22].
[1] L. V. Hau, S. E. Harris, Z. Dutton, and C. H. Behroozi, Nature (London) 397, 594 (1999).

[2] M. M. Kash, V. A. Sautenkov, A. S. Zibrov, L. Hollberg, G. R. Welch, M. D. Lukin, Y. Rostovtsev, E. S. Fry, and M. O. Scully, Phys. Rev. Lett. 82, 5229 (1999).

[3] D. Budker, D. F. Kimball, S. M. Rochester, and V. V. Yashchuk, Phys. Rev. Lett. 83, 1767 (1999).

[4] M. D. Stenner, D. J. Gauthier, and M. A. Neifeld, Phys. Rev. Lett. 94, 053902 (2005).

[5] H. Kang, G. Hernandez, and Y. Zhu, Phys. Rev. Lett. 93, 073601 (2004).

[6] A. V. Turukhin, V. S. Sudarshanam, M. S. Shahriar, J. A. Musser, B. S. Ham, and P. R. Hemmer, Phys. Rev. Lett. 88, 023602 (2002).

[7] P. C. Ku, F. Sedgwick, C. J. Chang-Hasnain, P. Palinginis, T. Li, H. Wang, S. W. Chang, and S. L. Chuang, Opt. Lett. 29, 2291 (2004).

[8] M. S. Bigelow, N. N. Lepeshkin, and R. W. Boyd, Phys. Rev. Lett. 90, 113903 (2003); E. Baldit, K. Bencheikh, P. Monnier, J. A. Levenson, and V. Rouget, ibid. 95, 143601 (2005); P. Wu and D. V. G. L. N. Rao, ibid. 95, 253601 (2005); Q. Yang, J. T. Seo, B. Tabibi, and H. Wang, ibid. 95, 063902 (2005).

[9] G. S. Agarwal and T. N. Dey, Phys. Rev. A 68, 063816 (2003); 73, 043809 (2006).

[10] S. Chu and S. Wong, Phys. Rev. Lett. 48, 738 (1982); L. J. Wang, A. Kuzmich, and A. Dogariu, Nature (London) 406, 277 (2000); A. Dogariu, A. Kuzmich, and L. J. Wang, Phys. Rev. A 63, 053806 (2001).

[11] M. S. Bigelow, N. N. Lepeshkin, and R. W. Boyd, Science 301, 200 (2003).

[12] G. S. Agarwal and T. N. Dey, Phys. Rev. Lett. 92, 203901
(2004).

[13] B. D. Clader, Q-Han Park, and J. H. Eberly, Opt. Lett. 31, 2921 (2006); B. D. Clader and J. H. Eberly, J. Opt. Soc. Am. B 24, 916 (2007).

[14] S. L. McCall and E. L. Hahn, Phys. Rev. 183, 82 (1969); see also Solitons, edited by R. K. Bullough and P. J. Caudrey (Springer-Verlag, New York, 1980).

[15] G. L. Lamb, Jr., Rev. Mod. Phys. 43, 99 (1971).

[16] G. M. Gehring, A. Schweinsberg, C. Barsi, N. Kostinski, and R. W. Boyd, Science 302, 895 (2006).

[17] A. E. Kaplan and P. L. Shkolnikov, Phys. Rev. Lett. 75, 2316 (1995); R. K. Bullough and F. Ahmad, ibid. 27, 330 (1971).

[18] This warrants generalization of the previous definitions of travel time [E. P. Wigner, Phys. Rev. 98, 145 (1955); J. Peatross, S. A. Glasgow, and M. Ware, Phys. Rev. Lett. 84, 2370 (2000); A. I. Talukder, T. Haruta, and M. Tomita, ibid. 94, 223901 (2005)]. For example, one is given by $\langle t\rangle_{\vec{r}}$ $=\int t S(\vec{r}, t) d t / \int S(\vec{r}, t) d t$, where $S(\vec{r}, t)$ is the Poynting vector of the pulse.

[19] A. Rahman and J. H. Eberly, Phys. Rev. A 58, R805 (1998).

[20] N. Tan-no, K. Yokoto, and H. Inaba, Phys. Rev. Lett. 29, 1211 (1972); G. J. Salamo, H. M. Gibbs, and G. G. Churchill, ibid. 33, 273 (1974).

[21] For consistency we have checked numerically propagation of finite pulses in an inhomogeneously broadened gain medium, and recover results in agreement with the McCall-Hahn area theorem [14].

[22] G. S. Agarwal and J. H. Eberly, Phys. Rev. A 61, 013404 (2000); P. K. Panigrahi and G. S. Agarwal, ibid. 67, 033817 (2003). 